\title{
COVID-19-Specific Suicidal Thoughts and Behaviors in Psychiatrically Hospitalized Adolescents
}

\author{
Taylor A. Burke ${ }^{1,4}\left(\right.$ D $\cdot$ Alexandra H. Bettis ${ }^{3}$. Anastacia Kudinova ${ }^{1,2} \cdot$ Sarah A. Thomas ${ }^{1,4}$. Jacqueline Nesi ${ }^{1,4}$. \\ Leyla Erguder ${ }^{1,2}$. Heather A. MacPherson ${ }^{1,2}$. Elizabeth Thompson ${ }^{1,4}$. Brooke A. Ammerman ${ }^{5}$. Jennifer C. Wolff ${ }^{1,4}$
}

Accepted: 28 July 2021 / Published online: 6 August 2021

(c) The Author(s), under exclusive licence to Springer Science+Business Media, LLC, part of Springer Nature 2021

\begin{abstract}
This study examined the presence and correlates of COVID-specific suicidal thoughts and behaviors (i.e., thoughts of or engaging in intentional COVID-19 exposure with associated suicidal intent) among psychiatrically hospitalized adolescents. Adolescents $(\mathrm{N}=143)$ completed study measures as part of the standard intake process between March 13th and August 14th, 2020. Participants answered questionnaires assessing COVID-specific passive and active suicidal ideation (SI) and suicidal behavior, as well as COVID-related stressors and emotions, and public health guidance compliance. Findings highlights that COVID-specific SI is common in high-risk youth. COVID-specific SI was associated with COVID-19-related negative emotions, elevated stress, and decreased public health guidance compliance. Results suggest that COVID-specific suicidal thoughts and behaviors, and risk correlates, should be assessed within high-risk populations to facilitate prevention of risky behavior associated with intentional COVID-19 exposure.
\end{abstract}

Keywords COVID-19 $\cdot$ Suicidal ideation and behavior $\cdot$ Adolescents

The global COVID-19 pandemic has posed significant challenges to people's quality of life, putting stress on emotional, financial, academic, social, physical, and occupational wellbeing. Potentially related to this surge in stress, individuals have reported engaging in a number of COVID-19 related risky behaviors that, at their extreme, have been life-threatening. Indeed, an alarmingly high number of adults, upwards of $4.5 \%$, have reported exposing themselves to COVID-19 with associated suicidal intent [1]. This statistic is particularly concerning given that COVID-19 is highly contagious [2], thus any degree of intentional exposure could have a

Taylor A. Burke

taylor_burke@brown.edu

1 Department of Psychiatry and Human Behavior, Alpert Medical School of Brown University, Box G-BH700 Butler Drive, Providence, RI 02906, USA

2 Bradley Hospital, East Providence, USA

3 Department of Psychiatry and Behavioral Sciences, Vanderbilt University Medical Center, Nashville, USA

4 Rhode Island Hospital, Rhode Island, USA

5 Department of Psychology, University of Notre Dame, Notre Dame, USA meaningful impact on virus spread. These findings are in line with a small body of research that cites cases of enacting suicidal behavior through intentional disease exposure (i.e., to human immunodeficiency viruses [HIV]) [3-5]. This behavior has direct implications for increased suicide risk in the general population because access to potentially lethal means is associated with suicide deaths [6]. Thus, as potential exposure or access to the virus increases, intentional deaths due to COVID-19 may also increase.

The presence of intentional COVID-19 exposure with associated suicidal intent has critical public health implications; however, the sole study examining its prevalence was preliminary and conducted among a sample of onlinerecruited non-clinical adults [1]. Thus, the findings require replication as well as extension to youth, a population in which rates of suicidal behavior have drastically increased over the past decade [7], and which may be particularly affected by the unique stressors of the COVID-19 pandemic [8]. Indeed, recent evidence suggests that during the COVID-19 pandemic, the proportion of pediatric emergency department (ED) visits for mental health concerns increased significantly, particularly among youth over the age of 12 [9]. Findings also indicated that youth with mental health concerns presenting to the ED during the pandemic were 
significantly more likely to be hospitalized [9]. These alarming pediatric mental health trends underscore the importance of ascertaining whether the pandemic-specific mental health phenomenon of intentional COVID-19 exposure with associated suicidal intent is cause for concern. Replication of these findings is required among high-risk clinical populations, including those who require psychiatric hospitalization, as such populations may be at heightened risk for future selfinjurious behaviors [10].

Beyond evaluating whether prior findings of intentional COVID-19 exposure with suicidal intent (COVID-specific suicidal behavior) may be replicated among clinical youth, it is essential to examine the presence of COVID-specific suicidal ideation (SI). Indeed, SI typically precedes and is a prospective predictor of suicidal behavior [10]. Suicidal ideation is often considered to exist along a continuum of severity from passive SI (i.e., a desire to be dead) to active SI (i.e., a desire to kill oneself), with the former being more prevalent [11]. Although passive SI is often considered an indicator of lower clinical risk than active SI, recent metaanalytic evidence implicates passive SI as a predictor of suicidal behavior in its own right [11]. Thus, examining COVID-specific passive and active SI may provide greater insight into the prevalence of this phenomenon, as well as the future risk it poses. Further, it is vital to assess associated psychosocial and contextual factors of COVID-specific SI in high-risk youth to identify potential risk correlates. Prior work suggests that life stress may play a critical role in the onset and exacerbation of SI in youth [12-15]. Thus, youth who have experienced higher levels of stress (e.g., interpersonal or health-related) and negative emotions (e.g., anxiety, sadness) associated with the pandemic may be more prone to COVID-specific SI.

Prior research has suggested that COVID-specific suicidal thoughts and behaviors are associated with greater engagement in risk-taking behaviors in youth (e.g., drug use, risky sexual behavior) (e.g., [16-18]). Relatedly, the COVID-19 pandemic has presented an opportunity for youth to engage in risky behavior with respect to virus-exposure-disregarding public health guidelines, such as social distancing and wearing a mask. Thus, we sought to explore whether COVID-specific SI is associated with lower compliance with COVID-19 public health guidelines, representative of greater engagement in virus-exposure risk behavior.

\section{Current Study}

The primary aim of this exploratory study was to investigate the presence of suicidal behavior by means of intentional COVID-19 exposure, as well as related passive and active COVID-specific SI, among high-risk adolescents. This study also aimed to conduct exploratory analyses to identify factors that may be associated with COVID-specific passive and active SI, including COVID-related stressors and emotional responses. Finally, this study aimed to explore whether COVID-specific SI is associated with COVID-19 public health guideline compliance.

\section{Methods}

\section{Participants and Procedures}

Participants were 143 adolescents admitted to a psychiatric inpatient unit in the northeastern United States between March 13, 2020 and August 14, 2020. Adolescents were admitted to the unit due to risk of harming themselves and/ or others. All study questionnaires were completed as part of the standard intake process.

Participants ranged in age from 11 to $18(\mathrm{M}=15.13$; $\mathrm{SD}=1.74)$. Participants were $53.1 \%$ female, $44.8 \%$ male, and $2.1 \%$ intersex. Approximately $7.6 \%$ identified as transgender or gender queer, gender non-conforming, or gender fluid. Most participants (65.7\%) identified as White; $31.1 \%$ identified as Hispanic/Latinx, 26.6\% as Black, 1.4\% as Asian, $5.6 \%$ as American Indian or Alaska Native, and $16.1 \%$ as other race/ethnicity. $48.5 \%$ of the sample identified as heterosexual/straight, $28.8 \%$ as bisexual, $9.8 \%$ as homosexual, $3.8 \%$ as asexual, $4.5 \%$ as other sexual identity, and $4.5 \%$ preferred not to answer. The average length of stay on the inpatient unit was 9 days. See Table 1 for complete participant demographics.

The study was classified as retrospective chart review by the Institutional Review Board and a waiver of informed consent was approved.

\section{Measures}

\section{COVID-19-Specific Suicidal Behavior}

A single item assessed intentional exposure to COVID19: "Have you done anything to intentionally (on purpose) increase the chance that you would get COVID-19 (coronavirus)?" [1]. Participants who responded "yes" were asked several follow-up questions regarding reasoning for intentional exposure. One of these follow-up items was used to classify the intentional exposure as suicidal in nature: "Why did you intentionally (on purpose) do something to increase the chance that you would get COVID-19 (coronavirus)?" Participants were instructed to rate the following response, "I wanted to kill myself," on a 5-point Likert scale from 1 (Not at all) to 5 (Extremely). Participants who indicated any suicidal intent associated with the behavior (ratings 2-5) were coded as having engaged in COVID-specific suicidal behavior. 
Table 1 Study sample descriptive statistics

\begin{tabular}{|c|c|}
\hline & $\begin{array}{l}n(\%) \text { except } \\
\text { where noted }\end{array}$ \\
\hline Age, $M(\mathrm{SD})$ & $15.13(1.74)$ \\
\hline \multicolumn{2}{|l|}{ Biological sex } \\
\hline Female & $76(53.1)$ \\
\hline Male & $64(44.8)$ \\
\hline Intersex & $3(2.1)$ \\
\hline \multicolumn{2}{|l|}{ Gender } \\
\hline $\begin{array}{l}\text { Transgender, gender-queer, gender-non-conforming, } \\
\text { or gender fluid }\end{array}$ & $10(7.6)$ \\
\hline Prefer not to answer & $1(0.8)$ \\
\hline \multicolumn{2}{|l|}{ Race/Ethnicity } \\
\hline White, non-hispanic & $77(53.8)$ \\
\hline White, hispanic & $17(11.9)$ \\
\hline Black, non-hispanic & $18(12.6)$ \\
\hline Black, hispanic & $20(14)$ \\
\hline Asian & $2(1.4)$ \\
\hline American Indian or Alaska Native & $8(5.6)$ \\
\hline Native Hawaiian or other pacific islander & $0(0)$ \\
\hline Other & $23(16.1)$ \\
\hline Hispanic & $41(31.1)$ \\
\hline \multicolumn{2}{|l|}{ Sexual orientation } \\
\hline Heterosexual/straight & $64(48.5)$ \\
\hline Homosexual & $13(9.8)$ \\
\hline Bisexual & $38(28.8)$ \\
\hline Asexual & $5(3.8)$ \\
\hline Other sexual identity & $6(4.5)$ \\
\hline Prefer not to answer & $6(4.5)$ \\
\hline
\end{tabular}

$\mathrm{N}=143 ; 11$ participants are missing gender, sexual orientation, and ethnicity data and as such, valid $\%$ is presented

\section{COVID-19-Specific Passive and Active Suicidal Ideation}

A questionnaire developed for this study assessed COVID-specific SI, including two independent subscales assessing passive SI (i.e., desire to be dead) and active SI (i.e., desire to kill oneself). The passive SI subscale included four items (e.g., "I thought, 'I don't care if I am infected with COVID-19 because I don't care if I live or die."'); the active SI subscale included three items (e.g., "I thought about trying to get infected with COVID-19 so that it would kill me."). Items were rated from 1 (Not at all) to 5 (Extremely) and were summed to create subscale totals. Internal consistencies were high for the passive and active subscales $(\alpha=0.96, \alpha=0.90)$. A complete set of items for this questionnaire can be found in the supplementary materials (see Supplementary Table 1).

\section{COVID-19 Stressors}

Stressors associated with COVID-19 were assessed using an 11-item measure. Participants were asked, "To what degree did you experience any of the following stressors as a result of COVID-19 (coronavirus)?" Items were rated on a 5-point Likert scale from 1 (Not at all) to 5 (Extremely). Stressors were selected to assess multiple domains relevant to adolescents (e.g. [19, 20],) including interpersonal (family and peer), finances, health of self, and health of family members, with adaptations specific to COVID-19 (e.g., "Worried about getting COVID-19 (coronavirus) myself").

\section{COVID-19 Emotional Responses}

Emotions associated with COVID-19 were assessed by the question: "To what extent have you felt the following in relation to COVID-19 (coronavirus)?" All items were rated on a 5-point Likert scale from 0 (Not much or not at all) to 4 (A lot). Emotions were selected based on hypothesized relevance to experiences during COVID-19, including: sad, lonely, bored, anxious, angry, guilty, uncertain, and relaxed.

\section{Compliance with COVID-19 Public Health Guidelines}

Compliance with COVID-19 public health guidelines was assessed using a single item, "Overall, how often are you and your family following public health guidelines related to COVID-19 (e.g., washing hands, staying home, social distancing, staying six feet away from others)?" Response options were rated on a 5-point Likert scale from 1 (Never) to 5 (Always).

\section{Suicidal Ideation}

The Suicidal Ideation Questionnaire-Junior (SIQ-Jr; [21]) is a 15-item self-report measure developed to assess severity and frequency of SI in youth. Respondents specify how strongly each item applies to their experience on a 7-point Likert-type scale from 0 (I have never had this thought) to 6 (almost every day). The SIQ-Jr has been found to be a valid measure of youth SI [21] and demonstrated strong internal consistency $(\alpha=0.96)$ in the current study.

\section{Suicide Attempt History}

Lifetime history of suicide attempt was assessed using a single item adapted from the Self-Injurious Thoughts and Behaviors Interview (SITBI; [22]), "Have you ever made 
an actual suicide attempt, where you were trying to kill yourself, even just a little?" Response options were "Yes" or "No".

\section{Statistical Analyses}

Correlations and chi-square tests were used to examine the association between demographic variables and the severity of COVID-specific passive and active SI. To examine the validity of our COVID-specific passive and active SI measure, correlations were used to evaluate the association between the severity of COVID-specific passive and active SI and the severity of general SI. To further assess validity, $t$-tests were used to examine the association between the severity of COVID-specific passive and active SI and the presence versus absence of lifetime suicide attempt history.

A series of t-tests were used to examine the relationship between the presence of COVID-specific passive and active SI (dichotomized to assess presence versus absence of passive [presence indicated by sum $>4$ ] and active SI [presence indicated by sum $>3]$ ) and COVID-19 related factors (i.e., emotional responses, stressors, and compliance with COVID-19 public health guidelines). Hedges' $g$ was employed as a measure of effect size.

\section{Results}

A total of $2.1 \%(n=3)$ of participants in the larger sample reported intentionally exposing themselves to COVID-19, with two participants (1.4\%) reporting associated suicidal intent (i.e., COVID-specific suicidal behavior). A total of 143 youth completed the questionnaire assessing COVIDspecific suicidal behavior. The COVID-specific SI questionnaire was added to the standard intake questionnaire battery at a slightly later date than the COVID-specific suicidal behavior questionnaire and thus, a subset of the larger sample $(\mathrm{N}=143)$ completed this measure $(n=121)$. Of the subsample administered the COVID-specific SI questionnaire $(n=121), 38.8 \%(n=47)$ endorsed any COVIDspecific passive SI $(\mathrm{M}=6.95 ; \mathrm{SD}=4.81)$ and $23.1 \%$ $(n=28)$ endorsed any COVID-specific active SI $(\mathrm{M}=4.19$; $\mathrm{SD}=2.60)$. Females reported greater COVID-specific passive $(t=-2.62, p=0.010)$ and active $(t=-2.03, p=0.045)$ SI than males. COVID-specific passive and active SI did not differ based on age or ethnicity ( $p$ 's $>0.05$ ).

Scores on the SIQ-Jr were expectedly elevated with the mean score of $34.76(\mathrm{SD}=27.85)$ above the clinical threshold. Supporting the validity of the COVID-specific SI measure, both COVID-specific passive and active SI were moderately correlated with the SIQ-JR ( $r$ 's $=0.60$ and 0.45 , $p<0.001$, respectively). Participants with a lifetime history of SA reported significantly greater COVID-related passive $(\mathrm{t}=-2.81, p=0.006)$ and active $(\mathrm{t}=-3.01, p=0.003)$ SI.

See Table 2 for associations between the presence of COVID-specific SI and COVID-19-related emotional responses, stressors, and public health guideline compliance. Results suggested that both passive and active SI were associated with greater feelings of sadness, loneliness, anxiety, guilt, and uncertainty; passive SI was additionally associated with greater feelings of anger. Among COVID-19 stressors, both passive and active SI were associated with greater experience of financial problems. Whereas passive SI was additionally associated with lack of access to basic needs, active SI was associated with greater interpersonal conflict at home. Additionally, findings suggested that the experience of passive SI was associated with lesser compliance with COVID-19 public health guidelines; active SI was not associated with COVID-19 public health guideline compliance. ${ }^{1}$

\section{Discussion}

This exploratory study is the first to examine the phenomenon of COVID-specific suicidal thoughts and behaviors among youth. The present study highlights intentional exposure to COVID-19 as a potential means of suicidal behavior in psychiatrically hospitalized adolescents, and thus fodder for suicidal thoughts. While only $1.4 \%$ of youth endorsed engaging in COVID-specific suicidal behavior, $38 \%$ endorsed COVID-specific passive SI and 25\% endorsed COVID-specific active SI. These findings align with prior work in adults [1], supporting the role of COVID-19 in experiences of suicidal behavior, and extend these findings to SI.

Although in a community sample of adults, $9.2 \%$ reported intentional COVID-19 exposure and $4.5 \%$ intentional exposure with associated suicidal intent [1], only $2.1 \%$ of this adolescent sample reported intentional exposure, with $1.4 \%$ reporting it to be suicidal in nature. One reason for this discrepancy may be the initial media reports of COVID-19 rarely affecting children, which may lead to youth perceiving COVID-19 as less deadly. Such a perception is not inaccurate, as recent findings suggest adolescents have lower susceptibility to hospitalization and mortality from COVID-19 compared to adults [23-25]. It is possible, then, that access or exposure to such information about COVID-19 may have reduced the degree to which adolescents consider intentional COVID-19 exposure as means to die by suicide. It is also possible that youth may have less knowledge about infectious disease contagion as compared to adults, also

\footnotetext{
${ }^{1}$ We conducted post-hoc Benjamini-Hochberg procedure using a false discovery rate of 0.05 . All significant findings remained unchanged with this correction.
} 
Table 2 Associations between COVID-19-specific passive and active suicidal ideation and COVID-19-related emotional responses and stressors

\begin{tabular}{|c|c|c|c|c|c|c|c|c|}
\hline \multirow[t]{3}{*}{ Characteristic } & \multicolumn{6}{|c|}{ COVID-19 specific suicidal ideation type } & & \\
\hline & \multicolumn{4}{|c|}{ COVID-19 specific active suicidal ideation } & \multicolumn{4}{|c|}{ COVID-19 specific passive suicidal ideation } \\
\hline & $\mathrm{No} / \mathrm{M}(\mathrm{SD})$ & Yes/ M (SD) & $t$ value & $g$ & $\mathrm{No} / \mathrm{M}(\mathrm{SD})$ & Yes/ M (SD) & $t$ value & $g$ \\
\hline $\begin{array}{l}\text { COVID-19 emotional } \\
\text { responses }\end{array}$ & $\mathrm{n}=93$ & $\mathrm{n}=28$ & & & $\mathrm{n}=74$ & $\mathrm{n}=47$ & & \\
\hline Feeling sad & $1.63(1.36)$ & $2.25(1.40)$ & $-2.09(p=0.039)^{*}$ & 0.45 & $1.53(1.32)$ & $2.17(1.42)$ & $-2.54(p=0.012)^{* *}$ & 0.47 \\
\hline Feeling relaxed & $1.02(1.09)$ & $0.96(1.17)$ & $0.24(p=0.812)$ & 0.05 & $0.96(1.04)$ & $1.09(1.21)$ & $-0.61(p=0.545)$ & 0.12 \\
\hline Feeling lonely & $1.87(1.40)$ & $2.50(1.40)$ & $-2.08(p=0.039)^{*}$ & 0.45 & $1.73(1.39)$ & $2.47(1.37)$ & $-2.87(p=0.005)^{* *}$ & 0.53 \\
\hline Feeling bored & $2.68(1.25)$ & $2.86(1.21)$ & $-0.67(p=0.504)$ & 0.14 & $2.58(1.29)$ & $2.94(1.13)$ & $-1.54(p=0.125)$ & 0.29 \\
\hline Feeling anxious & $1.54(1.43)$ & $2.46(1.43)$ & $-3.01(p=0.003)^{* *}$ & 0.64 & $1.45(1.42)$ & $2.23(1.45)$ & $-2.96(p=0.004)^{* *}$ & 0.54 \\
\hline Feeling angry & $1.60(1.47)$ & $2.18(1.42)$ & $-1.84(p=0.069)$ & 0.40 & $1.49(1.45)$ & $2.13(1.44)$ & $-2.38(p=0.019)^{* *}$ & 0.44 \\
\hline Feeling guilty & $0.63(1.21)$ & $1.64(1.47)$ & $-3.66(p<0.001)^{* * *}$ & 0.79 & $0.45(0.95)$ & $1.53(1.59)$ & $-4.23(p<0.001)^{* * *}$ & 0.87 \\
\hline Feeling uncertain & $1.12(1.34)$ & $2.04(1.40)$ & $-3.14(p=0.002)^{* *}$ & 0.68 & $0.96(1.19)$ & $1.91(1.53)$ & $-3.64(p<0.001)^{* * *}$ & 0.71 \\
\hline \multicolumn{9}{|l|}{ COVID-19 stressors } \\
\hline $\begin{array}{l}\text { Lack of access to basic } \\
\text { needs }\end{array}$ & $1.38(0.79)$ & $1.93(1.12)$ & $-2.92(p=0.004)^{* *}$ & 0.63 & $1.42(0.83)$ & $1.64(1.01)$ & $-1.30(p=0.195)$ & 0.24 \\
\hline Money problems & $1.61(0.94)$ & $2.46(1.37)$ & $-3.07(p=0.004)^{* *}$ & 0.81 & $1.59(0.98)$ & $2.15(1.23)$ & $-2.61(p=0.011)^{* *}$ & 0.52 \\
\hline $\begin{array}{l}\text { Fighting/arguing/conflict } \\
\text { at home }\end{array}$ & $2.34(1.30)$ & $2.61(1.29)$ & $-0.94(p=0.348)$ & 0.21 & $2.15(1.22)$ & $2.81(1.31)$ & $-2.81(p=0.006)^{* *}$ & 0.52 \\
\hline $\begin{array}{l}\text { Cutoff from contact with } \\
\text { others }\end{array}$ & $2.32(1.31)$ & $2.71(1.51)$ & $-1.34(p=0.184)$ & 0.29 & $2.28(1.30)$ & $2.62(1.45)$ & $-1.31(p=0.192)$ & 0.25 \\
\hline $\begin{array}{l}\text { Unable to leave home } \\
\text { when desired }\end{array}$ & $2.70(1.28)$ & $3.04(1.40)$ & $-1.19(p=0.236)$ & 0.26 & $2.78(1.32)$ & $2.77(1.32)$ & $0.07(p=0.942)$ & 0.01 \\
\hline $\begin{array}{l}\text { Unable to attend special } \\
\text { events }\end{array}$ & $2.47(1.39)$ & $2.61(1.57)$ & $-0.43(p=0.665)$ & 0.10 & $2.49(1.41)$ & $2.53(1.47)$ & $-0.17(p=0.865)$ & 0.03 \\
\hline $\begin{array}{l}\text { Having to change who } \\
\text { living with }\end{array}$ & $1.46(0.92)$ & $1.75(1.18)$ & $-1.36(p=0.176)$ & 0.29 & $1.49(0.93)$ & $1.60(1.08)$ & $-0.59(p=0.554)$ & 0.11 \\
\hline $\begin{array}{l}\text { Unable to see people } \\
\text { cared about }\end{array}$ & $2.65(1.36)$ & $3.00(1.47)$ & $-1.19(p=0.238)$ & 0.25 & $2.66(1.38)$ & $2.83(1.42)$ & $-0.65(p=0.520)$ & 0.12 \\
\hline $\begin{array}{l}\text { Someone cared about } \\
\text { got sick }\end{array}$ & $1.63(1.15)$ & $2.00(1.25)$ & $-1.45(p=0.151)$ & 0.31 & $1.61(1.10)$ & $1.89(1.29)$ & $-1.30(p=0.195)$ & 0.24 \\
\hline $\begin{array}{l}\text { Worried about getting } \\
\text { COVID }\end{array}$ & $2.00(1.28)$ & $2.32(1.42)$ & $-1.14(p=0.257)$ & 0.24 & $2.14(1.32)$ & $1.98(1.31)$ & $0.64(p=0.525)$ & 0.12 \\
\hline $\begin{array}{l}\text { Worried about other get- } \\
\text { ting COVID }\end{array}$ & $2.66(1.36)$ & $3.21(1.52)$ & $-1.85(p=0.067)$ & 0.39 & $2.65(1.37)$ & $3.00(1.47)$ & $-1.34(p=0.184)$ & 0.25 \\
\hline $\begin{array}{l}\text { Social distancing com- } \\
\text { pliance }\end{array}$ & $3.98(1.17)$ & $3.21(1.40)$ & $2.63(p=0.012)^{* *}$ & 0.63 & $3.95(1.25)$ & $3.57(1.26)$ & $1.59(p=0.115)$ & 0.30 \\
\hline
\end{tabular}

$n=121 ; * p<.05 ; * * p<.01 ; * * * p<.001 ; g=$ Hedges' $g$

contributing to lower rates of intentional exposure compared to prior research in adults.

We found that over one-third of psychiatrically hospitalized adolescents have experienced recent COVID-specific passive SI, reflecting a general ambivalence towards contracting and/or dying by COVID-19. Moreover, one-quarter of youth have experienced recent COVID-specific active SI, reflecting potentially more serious and less prevalent thoughts about actively engaging in a behavior to contract the virus in an effort to die. Despite the concept of COVIDspecific SI being new, requiring further validation of items to assess this construct, COVID-specific passive and active SI were associated with lifetime history of suicide attempts and demonstrated moderate correlations with the SIQ-Jr, a well-established measure of general SI in adolescents. These findings provide some evidence of construct validity for our measure and highlight that COVID-19 may represent a new context through which youth with histories of SI or suicidal behavior may experience suicidal thoughts. However, given that correlations with a measure of general SI were only moderate, our findings suggest COVID-specific SI may be a distinct construct worthy of future study. Further research is needed to explore the prevalence of COVID-specific SI in a community sample and to evaluate whether the frequency and severity of these thoughts may shift over the course of this ongoing, dynamic pandemic. 
Results have important implications for assessing suicide risk in psychiatrically hospitalized adolescents. First, findings highlight the importance of directly assessing the link between suicidality and COVID-19 to ascertain whether youth are thinking about engaging in intentional COVID-19 exposure with suicidal intent, or whether youth are experiencing general SI secondary to COVID-related stress. Second, results demonstrated associations between COVID-specific SI and COVID-related negative emotions and stressors, particularly economic stress. The observed relationships between stress and COVID-specific SI may indicate potential treatment targets, such as skills specific to managing stress that may have been exacerbated in the pandemic. Relatedly, this preliminary study highlights the need for expanded social services to support families whose financial wellbeing has been impacted by COVID-19.

Findings also have public health implications for managing exposure to and spread of COVID-19 given that COVIDspecific SI was associated with lower likelihood of following COVID-19 public health guidelines. Although the rate of COVID-19-specific suicidal behavior was relatively low $(1.4 \%)$ in our sample, it is possible that COVID-specific SI may influence greater noncompliance with COVID-19 guidelines (e.g., social distancing, mask wearing), and thus indirectly influence greater COVID-19 exposure. Given epidemiological evidence for the reliance of COVID-19 spread pattern on frequently asymptomatic 'superspreaders', this could incur increased risk and virus spread not only for vulnerable adolescents, but also for the general population [26-28]. Additional research is needed to disentangle the relationships between the COVID-specific SI and COVID19 risk behaviors in adolescents.

The present study has implications beyond those tied to the COVID-19 pandemic in that it can inform preventative measures to take in the case of future epidemics or pandemics. For example, our work indicates that it will be important to directly assess SI related to any future communicable diseases as well as to assess access to such diseases, in order to appropriately inform safety planning measures. Furthermore, the present findings shed light on the necessity of clinicians and researchers alike to keep apprised of naturally occurring, large-scale changes in the availability of lethal means, as the emergence of new lethal means may have an impact on the prevalence, form, and function of suicidal ideation and behavior.

The current exploratory study has a number of limitations that must be considered. First, this study was limited by the use of cross-sectional, self-report measures. Second, the relatively small sample limits our ability to explore time effects; it is possible that findings may differ across pandemic stages. Third, due to the urgency of the need to assess COVID-related mental health outcomes in youth, our COVID-related measures have not been validated. Although the COVID-related emotional responses and stress measures utilized in this study have high face validity, they have not been psychometrically evaluated. Fourth, our measure of COVID-19 public health guideline compliance conflates the behavior of the adolescent and their family. Continued refinement of developmentally appropriate, COVID-related mental health measures is necessary to better understand the public health effects of this pandemic on adolescents and, most urgently, those at high risk for suicide. Additionally, the present study explored COVID-specific SI and suicidal behavior among psychiatrically hospitalized adolescents and thus results may not be generalizable to non-psychiatric samples. Finally, there is a risk of false positive results given the numerous exploratory analyses; we therefore underscore the need to conduct future studies with larger samples to ensure replicability and generalizability.

\section{Conclusion}

Our findings indicate that COVID-specific SI is common among psychiatrically hospitalized youth and is associated with COVID-related negative emotions, elevated stress, and decreased likelihood of following COVID-19 public health guidelines. Results underscore the importance of assessing the pandemic-related impact on mental health and suicide risk in vulnerable adolescents and have critical implications for managing exposure to and spread of COVID-19.

\section{Summary}

This study examined the presence and correlates of COVIDspecific suicidal thoughts and behaviors (i.e., thoughts of or engaging in intentional COVID-19 exposure with associated suicidal intent) among psychiatrically hospitalized adolescents. A total of 143 psychiatrically hospitalized adolescents completed study measures as part of the standard intake process between March 13th and August 14th, 2020. Participants answered questionnaires assessing COVIDspecific passive and active SI and suicidal behavior, as well as COVID-related stressors and emotions, and public health guidance compliance. Approximately $1.4 \%$ of the sample reported COVID-specific suicidal behavior, 38.8\% experienced COVID-specific passive SI, and $23.1 \%$ experienced COVID-specific active SI. COVID-specific SI was associated with COVID-related negative emotions, elevated stress, and decreased public health guidance compliance. Limitations include the cross-sectional nature of the study as well as its reliance on self-report. Results suggest that COVID-specific SI is common in high-risk youth. COVIDspecific suicidal thoughts and behaviors, and risk correlates, should be assessed within high-risk populations to facilitate 
prevention of risky behavior associated with intentional COVID-19 exposure. Such prevention is integral to protecting youth and preventing COVID-19 spread.

Supplementary Information The online version contains supplementary material available at https://doi.org/10.1007/s10578-021-01225-3.

Acknowledgements Taylor A. Burke [T32 MH019927], Alexandra H. Bettis [K23MH122737], Anastacia Kudinova [K23MH122587], and Jacqueline Nesi [K23MH122669] were supported by the National Institutes of Mental Health. Sarah A. Thomas was supported in part by Institutional Development Award Number U54GM115677 from the National Institute of General Medical Sciences of the National Institutes of Health, which funds Advance Clinical and Translational Research (Advance-CTR), and the National Institute on Drug Abuse [K23DA050911]. NIH had no role in the design and conduct of the study; collection, management, analysis, and interpretation of the data; and preparation, review, or approval of the manuscript; or decision to submit the manuscript for publication. The content is solely the responsibility of the authors and does not necessarily represent the official views of NIH.

Funding This research did not receive any specific grant from funding agencies in the public, commercial, or not-for-profit sectors.

\section{Declarations}

Conflict of interest The author declares that they have no conflict of interest.

Ethical Approval All procedures performed in studies involving human participants were in accordance with the ethical standards of the institutional and/or national research committee and with the 1964 Helsinki declaration and its later amendments or comparable ethical standards. For this type of study formal consent is not required.

\section{References}

1. Ammerman BA, Burke TA, Jacobucci R, McClure K (2021) Preliminary investigation of the association between COVID-19 and suicidal thoughts and behaviors in the U.S. J Psychiatr Res 134:32-38. https://doi.org/10.1016/j.jpsychires.2020.12.037

2. World Health Organization Coronavirus disease (COVID-19) Pandemic. In: 2020. https://www.who.int/emergencies/diseases/ novel-coronavirus-2019. Accessed 30 Dec 2020

3. Tompkins-Rosenblatt P (1997) Intentional HIV contraction: implications for direct child and youth care. Resid Treat Child Youth. https://doi.org/10.1300/J007v14n04_02

4. Frances RJ, Wikstrom T, Alcena V (1985) Contracting AIDS as a means of committing suicide. Am. J. Psychiatry 142

5. Papathomopoulos E (1989) Intentional infection with the aids virus as a means of suicide. Couns Psychol Q. https://doi.org/10. 1080/09515078908256665

6. Marzuk PM, Leon AC, Tardiff K et al (1992) The effect of access to lethal methods of injury on suicide rates. Arch Gen Psychiatry 49:451-458. https://doi.org/10.1001/archpsyc.1992.0182006003 1005

7. Curtin SC (2020) State suicide rates among adolescents and young adults aged 10-24: United States, 2000-2018. Natl Vital Stat Reports
8. Tang S, Xiang M, Cheung T, Xiang YT (2021) Mental health and its correlates among children and adolescents during COVID-19 school closure: the importance of parent-child discussion. J Affect Disord. https://doi.org/10.1016/j.jad.2020.10.016

9. Krass P, Dalton E, Doupnik SK, Esposito J (2021) US pediatric emergency department visits for mental health conditions during the COVID-19 pandemic. JAMA Netw. Open 4

10. Franklin JC, Ribeiro JD, Fox KR et al (2017) Risk factors for suicidal thoughts and behaviors: a meta-analysis of 50 years of research. Psychol Bull. https://doi.org/10.1037/bul0000084

11. Liu RT, Bettis AH, Burke TA (2019) Characterizing the phenomenology of passive suicidal ideation: A systematic review and meta-analysis of its prevalence, psychiatric comorbidity, correlates, and comparisons with active suicidal ideation. Psychol Med

12. Grover KE, Green KL, Pettit JW et al (2009) Problem solving moderates the effects of life event stress and chronic stress on suicidal behaviors in adolescence. J Clin Psychol 65:1281-1290. https://doi.org/10.1002/jclp.20632

13. Pettit JW, Green KL, Grover KE et al (2011) Domains of chronic stress and suicidal behaviors among inpatient adolescents. J Clin Child Adolesc Psychol 40:494-499. https://doi.org/10.1080/15374 416.2011.563466

14. Miller AB, Eisenlohr-Moul T, Giletta M et al (2017) A withinperson approach to risk for suicidal ideation and suicidal behavior: examining the roles of depression, stress, and abuse exposure. $\mathbf{J}$ Consult Clin Psychol 85:712-722. https://doi.org/10.1037/ccp00 00210

15. MacKin DM, Perlman G, Davila J et al (2017) Social support buffers the effect of interpersonal life stress on suicidal ideation and self-injury during adolescence. Psychol Med 47:1149-1161. https://doi.org/10.1017/S0033291716003275

16. Ammerman BA, Steinberg L, McCloskey MS (2018) Risk-taking behavior and suicidality: the unique role of adolescent drug use. $\mathrm{J}$ Clin Child Adolesc Psychol 47:131-141. https://doi.org/10.1080/ 15374416.2016.1220313

17. Pena JB, Matthieu MM, Zayas LH et al (2012) Co-occurring risk behaviors among white, black, and hispanic US high school adolescents with suicide attempts requiring medical attention, 1999-2007: Implications for future prevention initiatives. Soc Psychiatry Psychiatr Epidemiol 47:29-42. https://doi.org/10.1007/ s00127-010-0322-z

18. Sullivan CJ, Childs KK, O'Connell D (2010) Adolescent risk behavior subgroups: an empirical assessment. J Youth Adolesc 39:541-562. https://doi.org/10.1007/s10964-009-9445-5

19. Rudolph KD, Hammen C (1999) Age and gender as determinants of stress exposure, generation, and reactions in youngsters: a transactional perspective. Child Dev 70:660-677. https://doi.org/ 10.1111/1467-8624.00048

20. Hammen C, Rudolph K, Weisz J et al (1999) The context of depression in clinic-referred youth: neglected areas in treatment. J Am Acad Child Adolesc Psychiatry 38:64-71. https://doi.org/ 10.1097/00004583-199901000-00021

21. Reynolds WM, Mazza JJ (1999) Assessment of suicidal ideation in inner-city children and young adolescents: reliability and validity of the suicidal ideation questionnaire-JR. School Psych Rev 28:17-30

22. Nock MK, Holmberg EB, Photos VI, Michel BD (2007) Self-injurious thoughts and behaviors interview: development, reliability, and validity in an adolescent sample. Psychol Assess 19:309-317. https://doi.org/10.1037/1040-3590.19.3.309

23. Bixler D, Miller AD, Mattison CP, et al (2020) SARS-CoV-2-associated deaths among persons aged $<21$ years - United States, February 12-July 31, 2020. MMWR Morb Mortal Wkly Rep 69:1324-1329. https://doi.org/10.15585/mmwr.mm6937e4

24. Kim L, Whitaker M, O'Halloran A, et al (2020) Hospitalization rates and characteristics of children aged $<18$ years hospitalized 
with laboratory-confirmed COVID-19 - COVID-NET, 14 States, March 1-July 25, 2020. MMWR Morb Mortal Wkly Rep 69:10811088. https://doi.org/10.15585/mmwr.mm6932e3

25. Viner RM, Mytton OT, Bonell C et al (2020) Susceptibility to SARS-CoV-2 infection among children and adolescents compared with adults: a systematic review and meta-analysis. JAMA Pediatr. https://doi.org/10.1001/jamapediatrics.2020.4573

26. Kenyon C (2020) The prominence of asymptomatic superspreaders in transmission mean universal face masking should be part of COVID-19 de-escalation strategies. Int. J. Infect. Dis. IJID Off Publ Int Soc Infect Dis 97:21-22

27. Asadi S, Wexler AS, Cappa CD et al (2019) Aerosol emission and superemission during human speech increase with voice loudness. Sci Rep 9:2348. https://doi.org/10.1038/s41598-019-38808-Z
28. Lloyd-Smith JO, Schreiber SJ, Kopp PE, Getz WM (2005) Superspreading and the effect of individual variation on disease emergence. Nature 438:355-359. https://doi.org/10.1038/nature04153

Publisher's Note Springer Nature remains neutral with regard to jurisdictional claims in published maps and institutional affiliations. 